\title{
The Use of Echocardiography in Pediatric Interventional Cardiac Catheterization Procedures
}

\author{
ACHI LUDOMIRSKY, M.D. \\ From the Division of Pediatric Cardiology, C.S. Mott Children's Hospital, University of Michigan, Ann Arbor, Michigan
}

\begin{abstract}
The last decade saw a dramatic change in the management of congenital heart defects. The introduction and development in echocardiography and Doppler modalities enable definitive diagnosis of congenital lesions without cardiac catheterization. At the same time, new therapeutic procedures for congenital defects using the catheter as a channel for different procedures were developed. These therapeutic procedures have replaced cardiac surgery in many types of defects. The new developments in echocardiography and Doppler modalities provide accurate imaging and visualization of the transcatheter devices and brought these two imaging modalities into a close marriage. The use of echocardiography and Doppler in the catheterization laboratory became a must, especially when used in atrial (ASD) and ventricular septal defect (VSD) closure. In this article we will detail the role of echocardiography in the different transcatheter interventional procedures in the catheterization laboratory. Those include atrial septostomy, blade atrial septectomy, balloon dilation, ASD closure, VSD closure, patent ductus anterior closure, and pericardiocentesis. (J Interven Cardiol 1995;8;569-578)
\end{abstract}

\section{Introduction}

During the last decade, enormous progress was achieved in the area of interventional procedures performed with a catheter. These procedures include maneuvers to create intracardiac shunts by balloon atrial septostomy and blade septectomy as well as procedures that close intra- and extracardiac communications, and open obstructions in valves and vessels. The capability of performing these procedures with a catheter enhances and improves the treatment of patients with congenital heart disease. During the same time, two-dimensional and Doppler ultrasound reached a level in which it is considered the preferable noninvasive diagnostic technique for congenital heart disease in children. Improvement of resolution, penetration, and image quality brought ultrasound to the leading edge of imaging modalities.

Three approaches are used in cardiac ultrasound: transthoracic; transesophageal; and intravascular. These three methods allow the sonographer to visual-

Address for reprints: Achi Ludomirsky, M.D., Pediatric Cardiology Department, 1500 East Medical Center Drive, Box 0204, Ann Arbor, MI 48109. Iax: (313) 936-9470. ize the heart through different channels giving the catheterizer the option to choose the preferable one. During the last decade, interventional catheterization and ultrasound "married each other" in order to achieve the highest level of accuracy and precision in these procedures. Ultrasound is being used to diagnose, guide, and evaluate immediate and late results of interventional procedures. This article describes the use of echocardiography in different interventional procedures.

\section{Creation of an Intracardiac Communication: Balloon Atrial Septostomy and Blade Atrial Septectomy}

Balloon atrial septostomy and blade atrial septectomy are well-known techniques which create an intraatrial communication in order to increase mixing or to provide an unobstructed right to left atrial shunt. ${ }^{1}$ Transthoracic and transesophageal echocardiography delineate the atrial septum both in the axial and lateral planes. Definition of the atrial septum should delineate 


\section{LUDOMIRSKY}

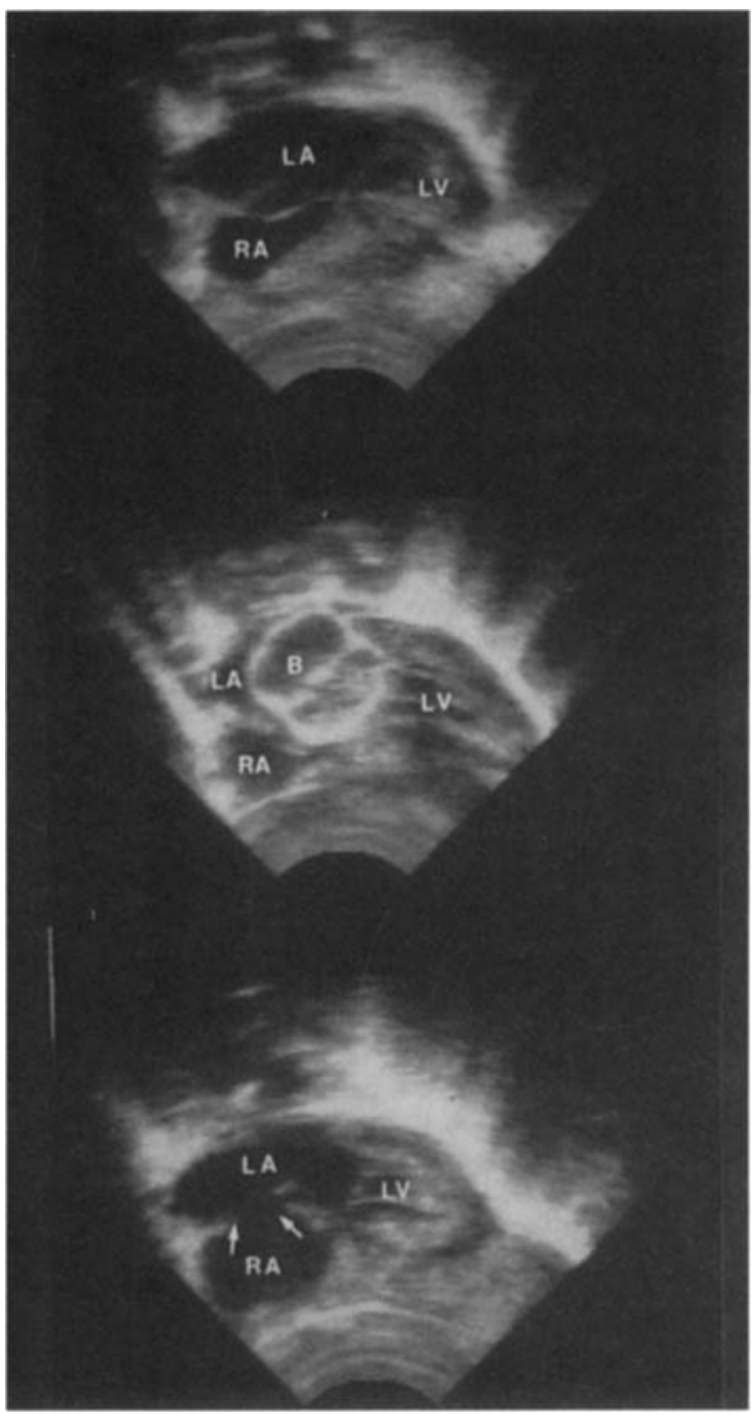

Figure 1. Two-dimensional transthoracic image from the subcostal view of a patient with transposition of the great arteries. Upper panel shows an intact atrial septum. Middle panel shows a balloon inflated in the left atrium. Lower panel shows the atrial septal defect (arrows) that was created by the procedure. $B=$ balloon; $\mathbf{L A}=$ left atrium; $\mathrm{LV}=$ left ventricle; $\mathrm{RA}=$ right atrium.

the existence of an atrial septum aneurysm or strings across the defect and a fenestration appearance. These factors can significantly affect the success of the interventional procedure. Visualization of the catheter course through the inferior vena cava or the superior vena cava into the right atrium and then across atrial septum to the left atrium is crucial. Once the catheter is identified in the right atrium, the echocardiographer

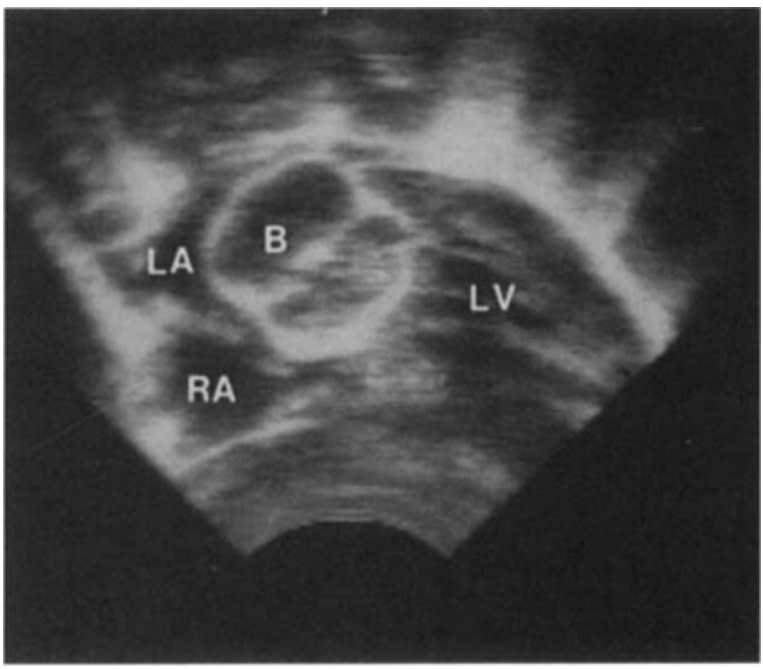

Figure 2. Two-dimensional transthoracic view of an inflated balloon in the left atrium. Note the proximity of the balloon to the mitral valve. $\mathrm{B}=$ balloon; $\mathrm{LA}=$ left atrium; $\mathrm{LV}=$ left ventricle; $\mathrm{RV}=$ right ventricle.

has to be sure that the catheter crosses the atrial septum into the left atrium and is not covered by septum primum. Using fluoroscopy alone can mislead and suggest that the catheter is already in the left atrium, but the existence of membrane covering the catheter is not visualized by this technique. Once the catheter is introduced into the left atrium, before inflating the balloon and/or opening the blade, ultrasound can help in localization of the tip of the catheter. The balloon must not be inflated if the tip of the catheter is situated either in one of the pulmonary veins or the mitral valve apparatus. Once the balloon is inflated or the blade is open, guidance of the procedure itself is possible. After crossing the atrial septum from left to right, the balloon should be deflated immediately and the blade should be closed and withdrawn (Figs. 1 and 2).

The immediate result of this procedure is evaluated by visualization of the atrial septum and ASD that was created. Color Doppler demonstrates the shunt and specifies the direction of the flow across the atrial septum. According to several authors, the ASD should exceed $45 \%$ of the total atrial septum diameter. ${ }^{2}$ Doppler evaluation of the shunt flow velocity can be used to demonstrate that the septal defect is nonrestrictive. Another important feature that indicates a successful procedure is free flail of the valve of the foramen ovale. Balloon septostomy with ultrasound guidance 
is being done at the bedside in several institutions. Our recommendation is that this procedure under ultrasound imaging should still be done in the catheterization lab, and immediate results and guidance should be provided by both invasive and noninvasive techniques.

\section{Balloon Dilation of Stenotic Valves and Coarctation on the Aorta}

Echocardiography in these conditions can provide the catheterizer with accurate anatomical and pathological details of the specific lesions. Accurate measurement of the valve annulus diameter in case of aortic or pulmonary valve stenosis is crucial for the selection of balloon size. The existence of a unicuspid or dysplastic valve in the aortic or pulmonary position may direct the catheterizer toward different approaches of treatment. Echocardiography should describe any possibility of subvalve or supravalve obstruction as an additional lesion. In the catheterization laboratory, ultrasound can identify if the catheters are centered across those valves before inflating the balloon. Immediate evaluation of residual obstruction and the amount of insufficiency caused by balloon dilation should be perlormed following the dilation procedure. The amount of valve insufficiency is evaluated by color flow and other Doppler techniques. In the case of cusp perforation or avulsion, ultrasound identifics the event immediately and accurate measurements should be taken.

In coarctation of the aorta, echocardiography should delineate the anatomy and differentiate between a discrete posterior shelf or long segment stenosis. When using transesophageal echocardiography imaging will measure the area of the coarctation by using the transverse and the longitudinal planes. Lately, the use of intervascular imaging during this procedure has improved the delineation of the diameter of the narrowest segment and the wall morphology. In several reports, a tear of the intima was seen immediately after balloon dilation of coarctation (Fig. 3). In some authors' opinion, an absence of a tear in the intima indicates a relative failure of this procedure. Follow-up studies showed that the majority of these tears heal without aortic aneurysm formation. Doppler techniques may be used in these cases in order to evaluate the pulsatility and distensibility of the descending aorta before and after this procedure. We have learned that both indices increased after a successful procedurc and can be used as predictors of success. ${ }^{3}$

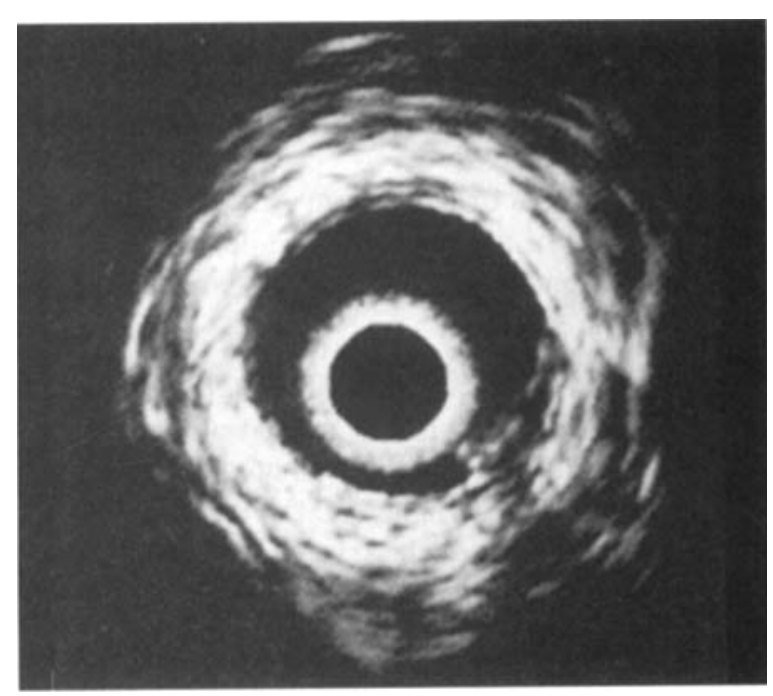

Figure 3. Transluminal view across the site of coarctation of the aorta after balloon dilation. Note the prominent tear of the intima.

\section{Closure of Shunt Lesions: Atrial Septal Defect, Ventricular Septal Defect, and Patent Ductus Arteriosus}

The evolution of transesophageal echocardiography opened a new window for cardiac imaging. The proximity of the esophagus to the left atrium facilitates the imaging of the posterior structures of the heart and provides an accurate visualization of all parts of the atrial septum.

Atrial Septal Defect Closure. Two devices are currently undergoing clinical investigations to close the secundum ASD: (1) the double umbrella device which attaches to the septum by two opposing umbrellas held against opposite sides of the septum by a spring mechanism; ${ }^{4}$ and (2) the button device. ${ }^{5}$ The metal components of these devices can be seen by fluoroscopy, but neither the ASD nor the atrial septum itself can be seen on fluoroscopy. The use of echocardiography is crucial for the following assessment of transcatheter ASD closure:

1. ASD sizing and visualization of the upper and lower septal rims.

2. Assessment of the ASD size by the balloon stretch technique.

3. Detection of leaks around the balloon by pulsed and color flow Doppler. 


\section{LUDOMIRSKY}

4. Device location relative to the left atrium/right atrium, and atrial appendages.

5. Proximity of the device to the mitral valve and tricuspid valve.

6. Identification of fenestrated ASDs.

7. Guidance of the release of the device.

8. Assessment of leaks across the device immediately after implantation.

Atrial septal defect visualization. One of the most critical aspects of ASD closure is the accurate determination of which defect will or will not be suitable for device closure. The current ASD device must be two times larger than the stretched size of the ASD. ${ }^{6}$ Transesophageal echocardiography has proven to be extremely useful, if not essential, in the imaging for the atrial septum for ASD measurement. ASDs with an unusually high or low position may require further evaluation. The atrial septum must have proper length and an adequate upper and lower rim to accommodate the total diameter of the device. The atrial septum is anatomically a concave-convex structure. For that reason, the imaging beam can cut the defect in different planes and not reflect the real size of the ASD. In the evaluation of atrial size, one should be aware of this limitation and try to document the largest diameter of the ASD by manipulating the probe anteriorly, posteriorly, as well as medially and laterally (Figs. 4 and $5)^{7}$

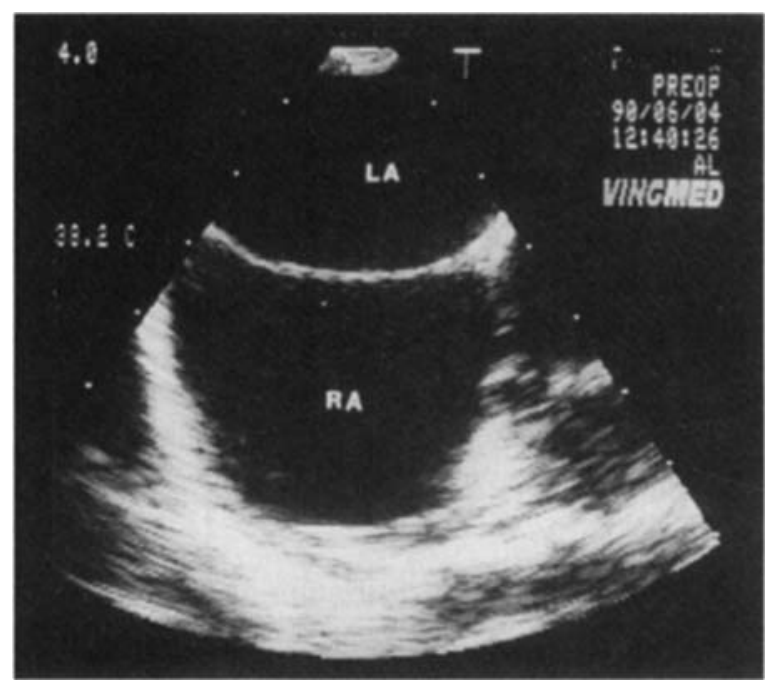

Figure 4. Transesophageal view of the atrial septum. Total length of atrial septum is visualized from the basal transesophageal plane. $\mathrm{LA}=$ left atrium; $\mathrm{RA}=$ right atrium.

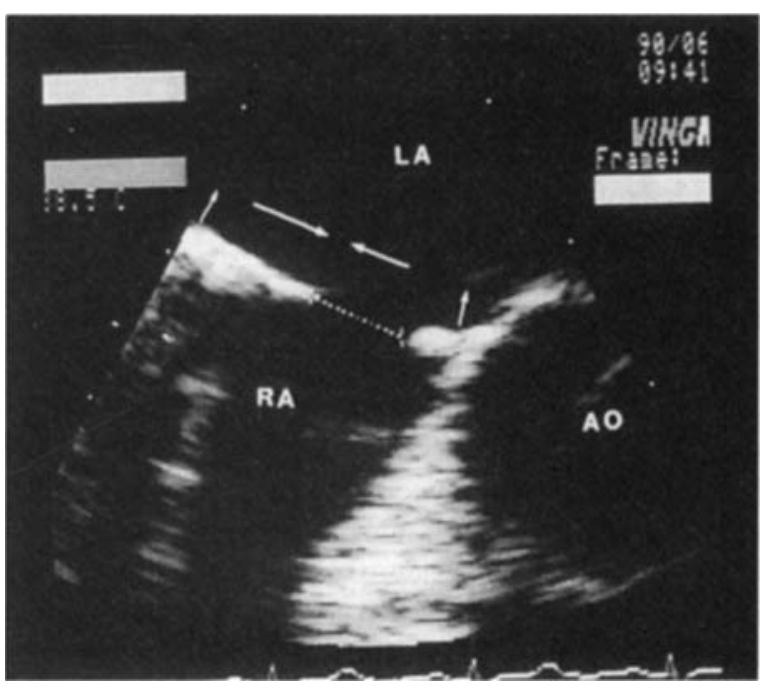

Figure 5. Transesophageal view of the atrial septum. Measurement of the total atrial septum (arrows), and defect (dotted line) sizes are demonstrated. $\mathrm{AO}=$ Aorta; $\mathrm{LA}=$ left atrium; $\mathrm{RA}=$ right atrium.

Balloon stretching technique for atrial septal defect sizing. ASD size is measured by the balloon sizing technique in all patients during cardiac catheterization. The balloon is well seen using fluoroscopy, but fluoroscopy does not allow the catheterizer to evaluate the location of the balloon within the atrial septum, or to visualize the upper and lower septal rims. Even though the balloon is "caught" by the atrial septum as it is pulled slowly from the left to the right atrium, transatrial shunts around the balloon can still exist and could mislead the catheterizer in the selection of the desirable device (Fig. 6). Pulsed and color flow Doppler can save the patient from unnecessary radiation and serve as a very sensitive modality for the detection of leaks across the sizing balloon. When a residual leak across the balloon is documented, the catheterizer is requested to inflate the balloon for a larger diameter until no residual leaks are visualized. ${ }^{8}$

Localization of the device. Transesophageal echocardiography assists in the localization of the tip of the sheath and/or catheter before the release of the device, and thereby prevents opening of the legs in the pulmonary veins or left atrial appendage or in proximity to the mitral valve. Because of the existence of the septum primum, the catheter may distort the septum toward the left atrium. The possibility of deploying the device near the septum primum on the right side of the septum creates a potential hazard 


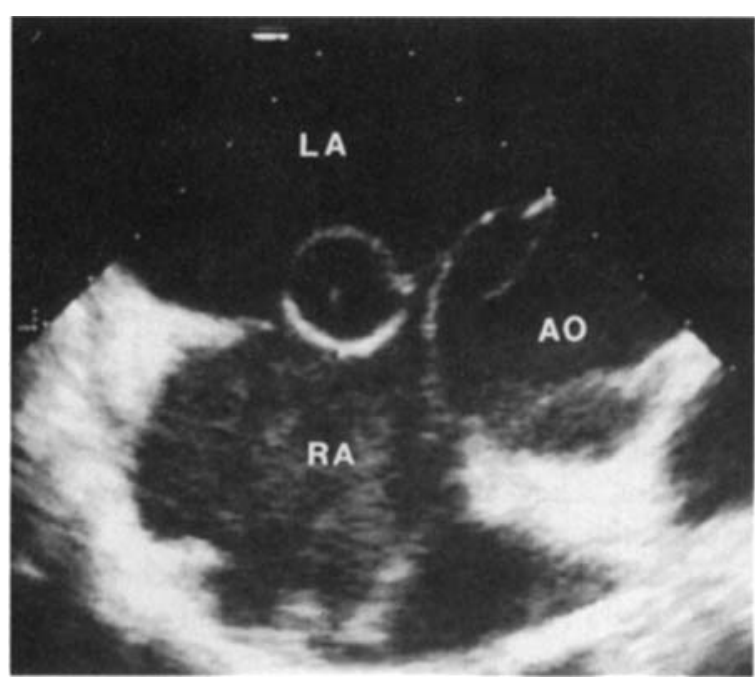

Figure 6. Transesophageal view of the atrial septum during balloon sizing of an ASD. The balloon is inflated in the left atrium and withdrawn across the atrial septal defect. $\mathrm{AO}=$ aorla; $\mathrm{LA}=$ left atrium; RA = right atrium.
(Fig. 7). Releasing the device close to the mitral and/or tricuspid valve may result in atrioventricular valve regurgitation. This should be assessed immediately by direct visualization and by color flow Doppler and the device position adjusted if necessary. It is important to visualize all eight legs of the device in the case of the Clamshell device (USCI, C.R. Bard, Billerica, MA, USA), and all six legs of the button device. This task is sometimes difficult because the legs may lie in different planes (Figs. 8 and 9). ${ }^{9}$

The fenestrated atrial septal defect. Transthoracic echocardiographic imaging of an ASD is sometimes limited by poor acoustic windows. Fenestrated ASDs introduce a major problem for transcatheter occlusion procedures. If a fenestrated ASD exists, the size of the actual defect could be underestimated (Fig. 10). The balloon stretching technique will not serve as an accurate measurement for the ASD if the balloon is trapped between the fenestration strands. In this case, the goal of the catheterizer will be to choose a device that will cover the whole ASD, including the fenestrated area.
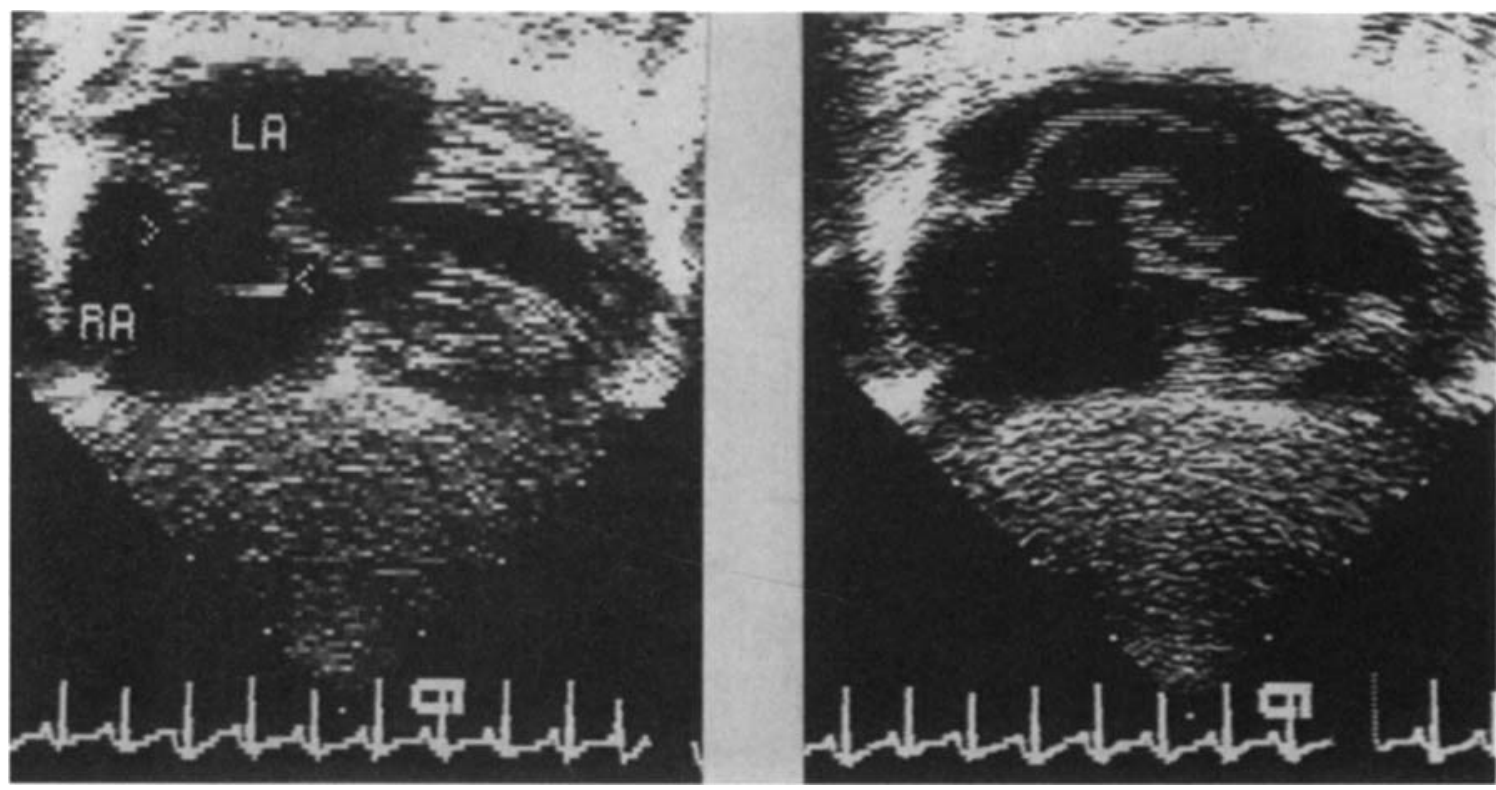

Figure 7. Two-dimensional transthoracic image from the subcostal vicw demonstrating an atrial septal defect and large aneurysm of the atrial septum. Note the septum primum can potentially cover the catheler tip in the right atrium. LA = left atrium; RA = right atrium. 


\section{LUDOMIRSKY}

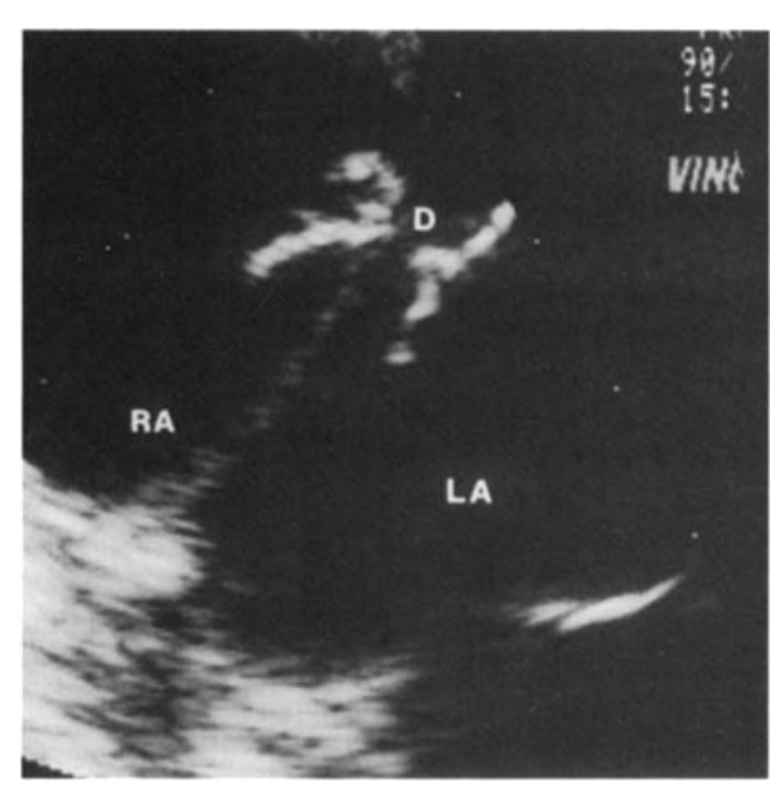

Figure 8. Transesophageal image of a clamshell device in an atrial septal defect. Note that only four legs are seen in this plane. LA = left atrium; RA = right atrium.
We have found that transesophageal echocardiography is essential for identification and management of fenestrated ASDs. ${ }^{10}$

Residual leaks across a device. Color flow Doppler is very sensitive in detection of residual leaks across ASD devices. Small residual leaks are often seen immediately after implantation, but in many cases, complete closure is obtained within 15 minutes. It is important to localize the residual leaks in order to guide further follow-up evaluation with both transthoracic and transesophageal echocardiography. If a large leak is present, a second device implantation is an option in the future.

Ventricular Septal Defect Closure. Echocardiography has a major role in the definition of the VSD and in the monitoring and guidance of its closure by a device. ${ }^{11}$ Each patient should be carefully evaluated by transthoracic echocardiography before entering the catheterization laboratory. In cases of apical muscular VSDs, the echocardiography visualization could be more accurate using the transthoracic approach than the transesophageal approach (Fig. 11). Transesopha-

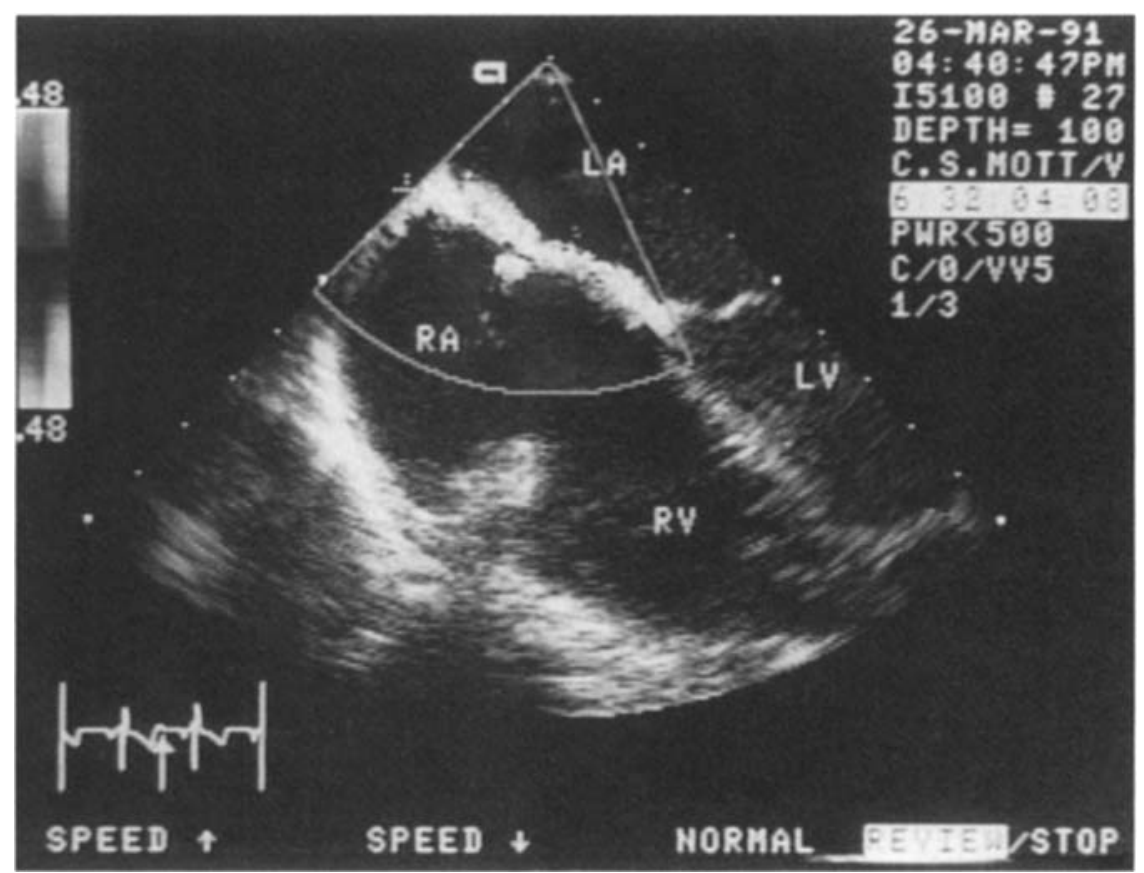

Figure 9. Transesophageal view of a button device in an atrial septal defect. Note that two legs are seen from this projection. $\mathrm{LA}=$ left atrium; $\mathbf{L V}=$ left ventricle; $\mathrm{RA}=$ right atrium; $\mathrm{RV}=$ right ventricle. 


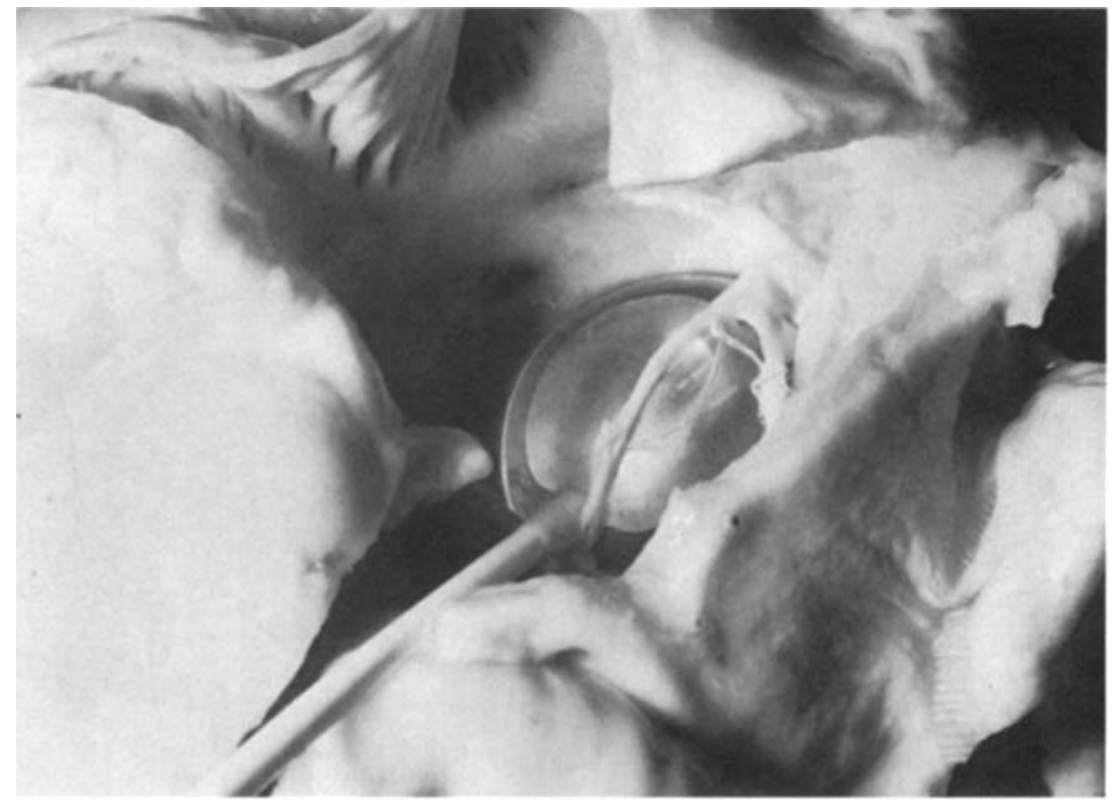

Figure 10. Pathological specimen of an atrial septal defect as viewed from the right atrium. There is a strand across the atrial septal defect. The balloon is trapped on the strand when withdrawn from left to right atrium.

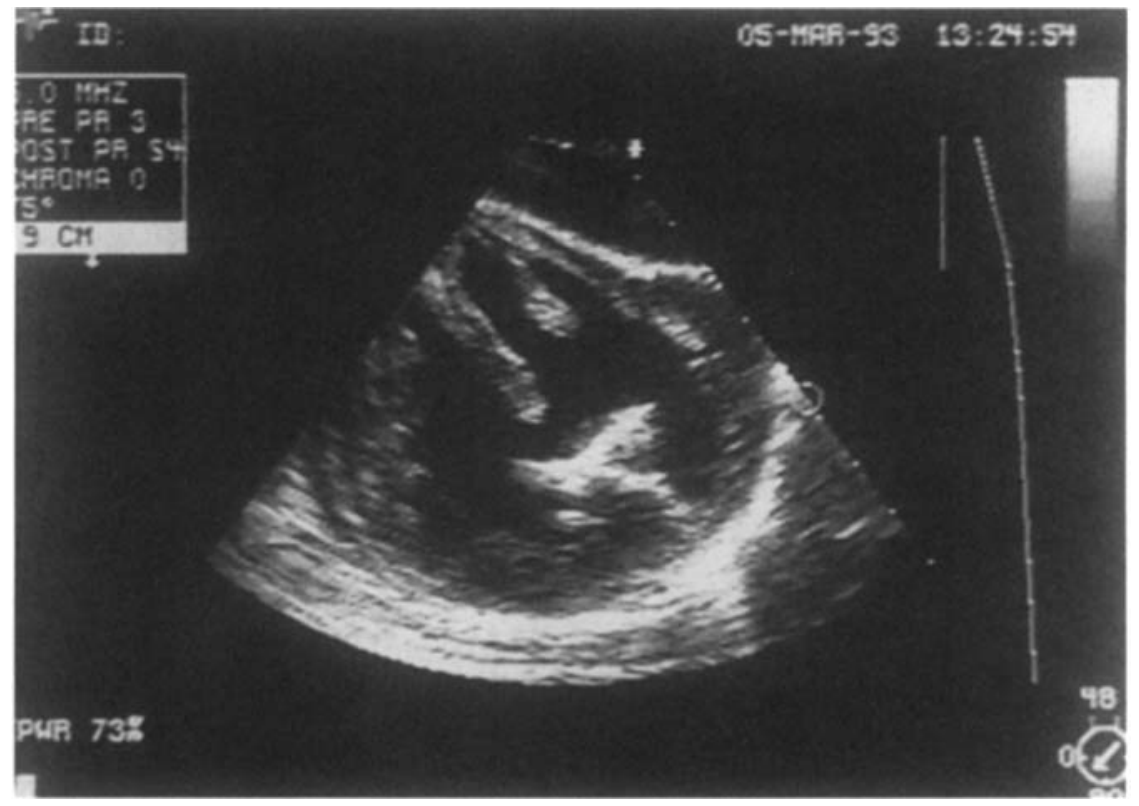

Figure 11. Transesophageal view of a device in the ventricular septum in a case of ventricular septal defect. Note the residual defect above the upper edge of the device. Only four legs are seen. 


\section{LUDOMIRSKY}

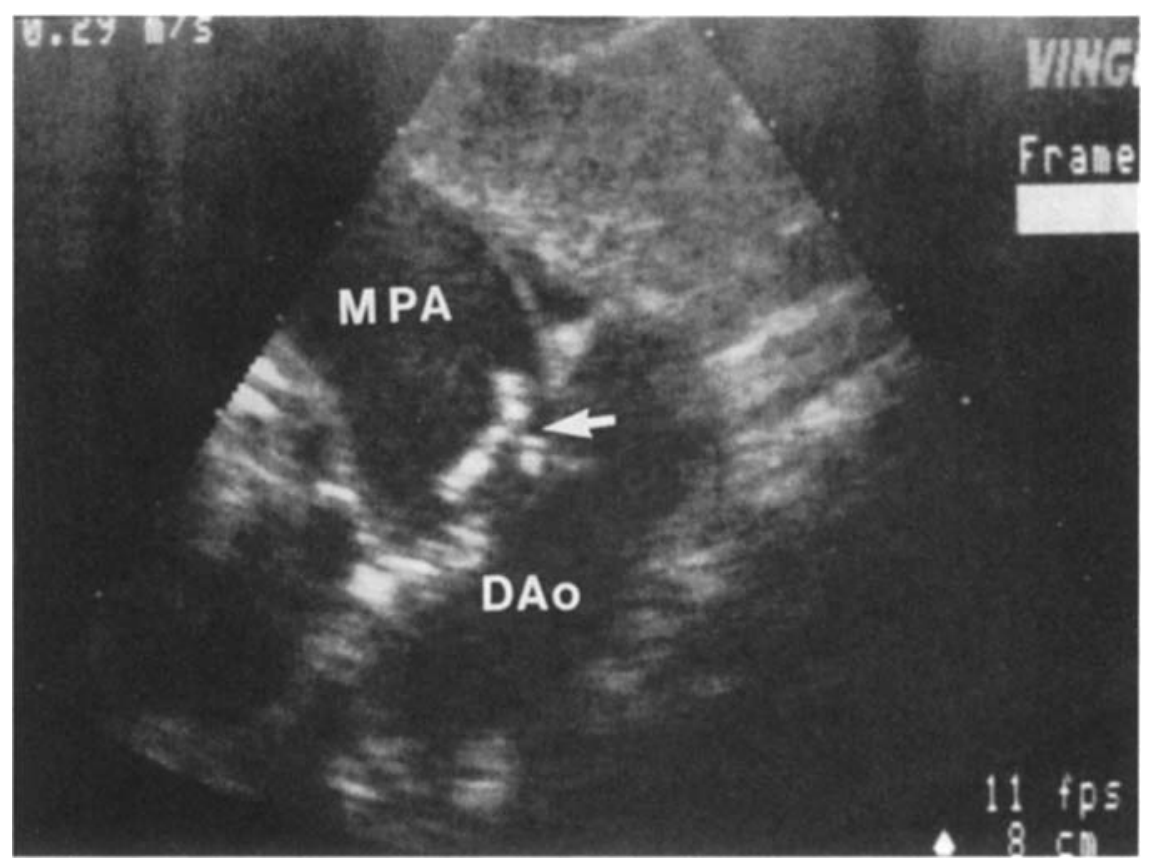

Figure 12. Two-dimensional transthoracic view from the high left parasternal view of a double umbrella device in a patent ductus arteriosus location. Visualization of the entire length of the device is possible. DA = descending aorta; MPA = main pulmonary artery.

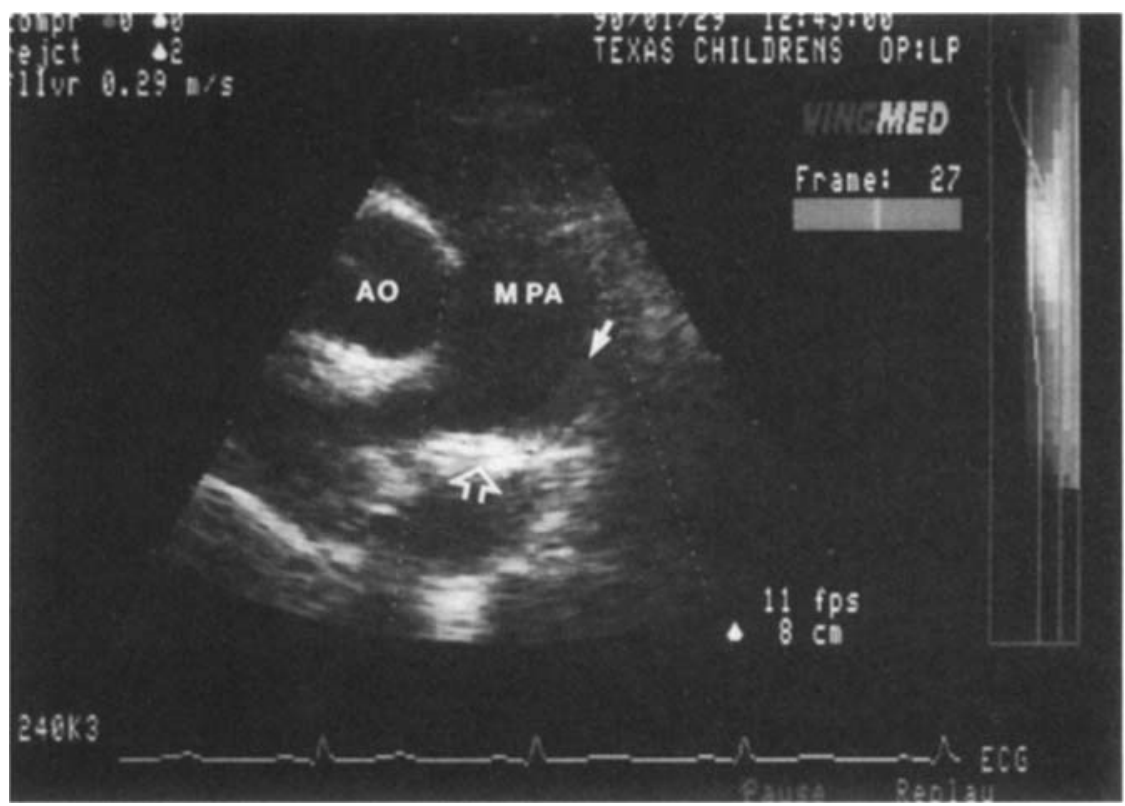

Figure 13. Two-dimensional and color flow Doppler display of a residual shunt (small arrow) across double umbrella device (large arrow). $\mathrm{AO}=$ aorta; $\mathrm{MPA}=$ main pulmonary artery. 


\section{ECHOCARDIOGRAPHY IN INTERVENTIONAL PROCEDURES}

geal echocardiography assists VSD closure in the following areas:

1. localization and sizing of the VSD

2. guiding different stages of the procedure including catheter and wirc manipulation through the VSD

3. assessment of the device location within the left ventricle/right ventricle for guidance of the release of the device

4. assessing proximity of the device to the atrioventricular valves

5. detection of residual leaks.

Patent Ductus Arteriosus Closure. The role of echocardiography in the transcatheter closure of the patent ductus arteriosus (PDA) is primarily in evaluation of anatomy and physiology before and after PDA closure. Identification, measuring, and localization of the ductus is helpful before the procedure takes place. Characterization of the ductus as elongated or funnel shape is helpful for the catheterizer. Doppler examination can determine if the PDA is restrictive. PDAs are closed either with an umbrella device or with coils. ${ }^{12,13}$ Using either technique, ultrasound should provide information regarding the presence of a residual shunt immediately postimplantation. There are two major types of leaks across the device: (1) low velocity jets across the foam or Dacron of the device; and (2) discrete high velocity jets through the upper or lower edges of these devices. We have learned that most of the slow velocity jets through the devices will disappear within 1.5-30 minutes after a procedure, while the high velocity discrete jets will either remain or disappear within 3-6 months. Both types of leaks can be identified by the transthoracic approach (Figs. 12 and 13$)^{14}$

\section{Pericardiocentesis}

Pericardiocentesis is done by introducing a needle or catheter into the pericardial sac and evacuating the pericardial fluid. Ultrasound is the best imaging modality to diagnose the existence, spread, location, and the amount of fluid. Using a transthoracic and/or transesophageal approach, the exact localization (i.e., anterior, posterior, circumferential, or localized) of the effusion can be made. Before the procedure is performed, the exact location of the effusion is critical for guiding the pericardiocentesis procedure. If needle position is unclear, a cautious maneuver can be taken by injecting effusion fluid back into the space in order to visualize bubbles in the pericardial sac. After evacuation of the fluid, repeat assessment of effusion size and ventricular function as well as possible atrial compromise should be performed. Doppler technique can identify very early alteration in diastolic ventricular function. This finding should disappear after successful evacuation of this fluid. In cases of emergency, this procedure can be done at the bedside, but our recommendation is to perform pericardiocentesis in the catheterization lab under direct visualization by echocardiography and fluoroscopy.

\section{Conclusion}

In general, with the improvement of available ultrasound modalities, (i.e., transesophageal transducers, miniaturization of devices) we envision more cooperation between cardiac catheterization and echocardiography. New echocardiographic modalities will enable us to perform on-line three-dimensional reconstruction in the near future. This technology together with the development of improved transluminal imaging will define echocardiography as a major participant in many pediatric interventional procedures in the future.

\section{References}

1. Rashkind WJ, Miller WW. Creation of an atrial septal defect without thoricotomy: A palliative approach to complete transposition of the great arteries. JAMA 1966;196:991-993.

2. Marantz. P, Huhta JC, Mullins CE, el al. Results of balloon valvuloplasty: Dysplastic versus typical pulmonary valve stenosis. JACC 1988:12(2):476-479.

3. Michelfelder E, Ludomirsky A, Lloyd TR, et al. Echocardjographic assessment of aortic compliance and distensibility before and after coarctation of the aorta repair. June 13-15, 1994. J Am Soc Echo 1994;7(3)2:S13.

4. Mullins CE. Therapeutic cardiac catheterization. In: Garson A, Bricker JT, McNamara DG, eds. The Science and Practice of Pediatric Cardiology. Philadelphia: Lea and Febiger, 1990 pp. 2183-2209.

5. Lloyd TR, Rao SP, Beekman RH III, et al. Atrial septal defect occlusion with the buttoned device (a multi-institutional trail U.S. trial). Am J Cardiol 1994;73:286-291.

6. Lock JE, Rome JJ, Davis R, et al. Transcatheter closure of atrial septal defects: Experimental studies. Circulation 1989; 79:1091-1099.

7. Hellenbrand WE, Fahey JT, McGowen FX, et al. Transesophageal echocardiographic guidance of transcatheter closure of atrial septal defect. Am J Cardiol 1990;66:207-213. 


\section{LUDOMIRSKY}

8. Morimoto K, Matsuzaki M, Tachma Y, et al. Diagnosis and quantitative evaluation of secundum-type atrial septal defect by transesophageal Doppler echocardiography. Am J Cardiol 1990;66:85-91.

9. Bridges ND, Newburger JW, Mayer JE, et al. Transcatheter closure of secundum ASD in pediatric patients: The first year's experience. (abstract) Am J Cardiol 1990;66:522.

10. Vick GW, Titus J. Defects of the atrial septum including the atrioventricular canal. In: Garson A, Bricker JT, McNamara DG, eds. The Science of Practice of Pediatric Cardiology. Philadelphia: Lea and Febiger, 1990, pp. $1023-1054$.
11. Lock JE, Block PC, McKay RC, et al. Transcatheter closure of ventricular septal defects. Circulation 1988;78(2):361-368.

12. O'Laughlin MP, Mullins CE. Transcatheter occlusion of ventricular septal defect. Cathet Cardiovase Diagn 1989;17: 175-179.

13. Lloyd TR, Fedderly R, Mendelsohn AM, et al. Transcatheter occlusion of patent ductus arteriosus with Gianturco coils. Circulation 1993;88(Part 1):1412-1420.

14. Ludomirsky A, O'Laughlin MP, Mullins CE. Color flow Doppler low-velocity enhancement in the assessment of residual leaks across patent ductus arteriosus occlusion devices. (abstract) JACC 1990;15(2):186A. 